\title{
Improvements Based on the Harris Algorithm
}

\author{
Huai Yang $\mathrm{Chen}^{1} \&$ Jinjie $C h n^{1}$
}

${ }^{1}$ College of Mechanical Engineering, University of Shanghai for Science and Technology, Shanghai, China

Correspondence: Huai yang Chen,College of Mechanical Engineering, University of Shanghai for Science and Technology, 334 Jun Gong Road, Shanghai 200093, China. Tel: 86-188-1758-3090. E-mail: m18817583090@163.com.

\author{
Received: May 5, 2013 Accepted: July 2, 2013 Online Published: August 21, 2013 \\ doi:10.5539/cis.v6n4p51 URL: http://dx.doi.org/10.5539/cis.v6n4p51
}

\begin{abstract}
Corner detection is a fundamental step in image processing, and it takes an important role in target tracking, image stitching and three-dimension reconstruction. Harris algorithm is widely used in corner detection for simple calculation and its detection result is not affected by image rotation and light intensity changes. Harris algorithm uses integral differential mask to extract the image gradient, and the edges information remains in the low frequency part of images. When dealing with images with a large number of edge information, integral differential weakens the low frequency part of images obviously, thus the detection result is not really good. Besides, Harris algorithm does not have the property of scale-invariant. For these reasons, fractional differential and multiple scale-space method are put forward in this article to improve Harris algorithm. Experiments show that the detection result of improved algorithm is better than original Harris algorithm in dealing with images of much detailed information.
\end{abstract}

Keywords: Harris algorithm, improved, fractional differential, multiple scale-space

\section{Introduction}

Harris corner detection algorithm was put forward by Chris Harris, Mi ke Stephens in 1988. Detailed steps of Harris algorithm are as follows:

\begin{tabular}{|c|c|c|}
\hline 1 & 1 & 1 \\
\hline 0 & 0 & 0 \\
\hline-1 & -1 & -1 \\
\hline
\end{tabular}

$X$

\begin{tabular}{|c|c|c|}
\hline 1 & 0 & -1 \\
\hline 1 & 0 & -1 \\
\hline 1 & 0 & -1 \\
\hline
\end{tabular}

$Y$

Figure 1.

The first step is to get the horizontal gradient and vertical gradient magnitude of each pixel in the image via convolution operations with Prewitt operator (Figure 1).

$$
\frac{\partial I}{\partial x}=I \otimes(-1,0,1), \frac{\partial I}{\partial y}=I \otimes(-1,0,1)
$$

The main purpose of second step is to reduce the noises in image. The algorithm selected Gaussian Window as image smoothing processing kernel. The following function is defined as:

$$
A=\left(\frac{\partial I}{\partial x}\right)^{2} \otimes w, B=\left(\frac{\partial I}{\partial x}\right)^{2} \otimes w, C=\left(\frac{\partial I}{\partial x} \times \frac{\partial I}{\partial y}\right) \otimes w, w=e^{-\left(u^{2}+v^{2}\right) / 2 \sigma^{2}}
$$


The third step is to calculate the corner response function of each pixel and determine the candidate points. Given one point $(x, y)$ on image $f$ and when it moves a small distance $u$ in the $X$ direction and $v$ in the $Y$ direction, gray-value changes is defined as:

$$
\begin{aligned}
& E_{u, v}(x, y)=\sum_{u, v} w_{u, v}[f(x+u, y+v)-f(x, y)]^{2}=\sum_{u, v} w_{u, v}\left\{u \frac{\partial f}{\partial x}+v \frac{\partial f}{\partial y}+O\left[u^{2}+v^{2}\right]\right\}^{2} \\
& \approx \sum_{u, v} w_{u, v}\left\{u \frac{\partial f}{\partial x}+v \frac{\partial f}{\partial y}\right\}^{2}
\end{aligned}
$$

$w_{u, v}$ represents the coefficient of Gaussian window in $(u, v)$, and $O$ means the symbol of infinitesimal.

The autocorrelation matrix $M$ is defined as:

$$
\begin{aligned}
& E_{u, v}(x, y)=\sum_{u, v} w_{u, v}[f(x+u, y+v)-f(x, y)]^{2}=\sum_{u, v} w_{u, v}\left\{u \frac{\partial f}{\partial x}+v \frac{\partial f}{\partial y}+O\left[u^{2}+v^{2}\right]\right\}^{2} \\
& \approx \sum_{u, v} w_{u, v}\left\{u \frac{\partial f}{\partial x}+v \frac{\partial f}{\partial y}\right\}^{2} \\
& M=\left(\begin{array}{ll}
A & C \\
C & B
\end{array}\right)=\left(\begin{array}{cc}
\left(\frac{\partial I}{\partial x}\right)^{2} \otimes w & \left(\frac{\partial I}{\partial x} \times \frac{\partial I}{\partial y}\right) \otimes w \\
\left(\frac{\partial I}{\partial x} \times \frac{\partial I}{\partial y}\right) \otimes w & \left(\frac{\partial I}{\partial y}\right)^{2} \otimes w
\end{array}\right)
\end{aligned}
$$

Image regions can be classified according to the eigenvalues $\left(\lambda_{1}, \lambda_{2}\right)$ of the autocorrelation $M$.

- $\lambda_{1} \approx \lambda_{2}$ and $\lambda_{1} \geq 0$. Both eigenvalues are big, and intensity variation is strong in all directions. This region is regarded as candidate corner.

- $\lambda_{1} \approx \lambda_{2}$ and $\lambda_{1} \approx 0$. Both eigenvalues are small, and the intensity variation is negligible in every direction. This region is considered to be plain.

- $\lambda_{1} \leq \lambda_{2}$ or $\lambda_{2} \leq \lambda_{1}$. One eigenvalue is big, and the other is small, and the intensity variation is strong in one direction. This region is thought to be edge.

The algorithm provides the following justification to measure the corner response function:

$$
R=\operatorname{Det}(M)-k T_{r}^{2}(M)
$$

$\operatorname{Det}(M)=\lambda_{1}+\lambda_{2}, T_{r}(M)=\lambda_{1}+\lambda_{2} \cdot k$ is usually adopted from 0.04 to 0.06 .

A point $(x, y)$ is selected as a corner if its corresponding response function value is greater than a given threshold $t_{1}$.

In order to improve the detection accuracy of Harris algorithm, many methods have been proposed. For example, a method of double-threshold was put forward to reduce false corners in detection result, and a multi-scale algorithm based on wavelet was presented to detect corners under different scales. Some researchers improved Harris algorithm using B spline curve, and so on. All these methods can improve Harris algorithm to some extent. This improvements proposed in this paper are based on fractional differential theory and the Gaussian theory, which is different from these methods presented above.

\section{Improved Algorithm}

\subsection{Fractional Differential Mask}

The purpose of this section is to introduce the basic theoretical background of fractional differential in signal processing and deduce the computation procedures of fractional differential mask.

The common definitions of fractional differential under Euclidean measurement are the Grünwald-Letnikov's definition, iemann-Liouville's definition and Caputo's definition. Among those definitions, Grünwald-Letnikov's definition is more easy to calculate because the only need coefficient is related to signal itself. Consequently, it is more applicable to image processing.

The generalized cognition of fractional differential in geometry is regard as fractional slope, which means the 
slope of its function. Similar to integral differential, fractional differential could enhance the high frequency part of the signal in some degree, but unlike integer order differential,while enhancing texture details in those areas that gray level does not change greatly, fractional differential can furthest preserve the low frequency part of the signal, and non-linearly keep high frequency edge areas that gray-value changes evidently. Fractional differential is better than integral differential in dealing with digital image with rich texture in theory.

Given that $f(x)$ represent an energy signal and its arguments satisfy Dirichlet's conditions, and its $v$-order Grünwald-Letnikov-based fractional differential is defined as:

$$
D_{G-L}^{v} f(x)=\left.\frac{d^{v}}{[d(x-a)]^{v}} f(x)\right|_{G-L}=\lim _{N \rightarrow \infty}\left\{\frac{\left(\frac{x-a}{N}\right)^{-v}}{\Gamma(-v)} \sum_{k=0}^{N-1} \frac{\Gamma(k-v)}{\Gamma(k+1)} \times f\left(x-k\left(\frac{x-a}{N}\right)\right)\right\}
$$

$v$ is any real number (including fraction), and the duration of signal $f(x)$ is $[a, x]$.

Suppose a $=0$ and divides the duration of $f(x)$ into $N$ equal parts, thus there are $N+1$ nodes. The $N+1$ causal pixels can describe as:

$$
\left\{\begin{array}{c}
f_{0} \equiv f(x) \\
f_{N-1} \equiv f(x / N) \\
\vdots \\
f_{N-1} \equiv f(x-k x / N) \\
f_{N} \equiv f(0)
\end{array}\right.
$$

When dealing with digital image, we can depose noncausal pixels by expanding the verge in a periodical way. Then (2.1.2) can be rewritten as:

$$
\left\{\begin{array}{c}
f_{0} \equiv f(x) \\
f_{-1} \equiv f(x+x / N) \\
\vdots \\
f_{-k} \equiv f(x+k x / N) \\
\vdots \\
f_{N} \equiv f(0)
\end{array}\right.
$$

When $N$ is big enough, (2.1.1) can be rewritten as:

$$
\left.\frac{d^{v}}{d x^{v}} f(x)\right|_{G-L} \cong \frac{x^{-v} N^{v}}{\Gamma(-v)} \sum_{k=0}^{N-1} \frac{\Gamma(k-v)}{\Gamma(k+1)} f\left(x-\frac{k x}{N}\right)=\frac{x^{-v} N^{v}}{\Gamma(-v)} \sum_{k=0}^{N-1} \frac{\Gamma(k-v)}{\Gamma(k+1)} f\left(x+\frac{v x}{2 N}-\frac{k x}{N}\right)
$$

(2.1.3) has introduced the signal value of $f(x)$ on non-node, and using Lagrange3-point interpolation expression to get better precision.the following expression is captured:

$$
\begin{aligned}
& f(\varepsilon) \cong \frac{\left(\varepsilon-x+\frac{k x}{N}\right)\left(\varepsilon-x+\frac{x}{N}+\frac{k x}{N}\right)}{2 x^{2} / N^{2}} f\left(x+\frac{x}{N}-\frac{k x}{N}\right)-\frac{\left(\varepsilon-x-\frac{x}{N}+\frac{k x}{N}\right)\left(\varepsilon-x+\frac{x}{N}+\frac{k x}{N}\right)}{x^{2} / N^{2}} f\left(x-\frac{k x}{N}\right)+ \\
& \frac{\left(\varepsilon-x-\frac{x}{N}+\frac{k x}{N}\right)\left(\varepsilon-x+\frac{k x}{N}\right)}{2 x^{2} / N^{2}} f\left(x-\frac{x}{N}-\frac{k x}{N}\right)
\end{aligned}
$$

Assume $\varepsilon=x+\frac{v x}{2 N}-\frac{k x}{N}$ and combine (2.14) with (2.1.5) we can get:

$$
\left.\frac{d^{v}}{d x^{v}} f(x)\right|_{G-L} \cong \frac{x^{-v} N^{v}}{\Gamma(-v)} \sum_{k=0}^{N-1} \frac{\Gamma(k-v)}{\Gamma(k+1)} \times f\left[f_{k}+\frac{v}{4}\left(f_{k-1}-f_{k+1}\right)+\frac{v^{2}}{8}\left(f_{k-1}-2 f_{k}+f_{k+1}\right)\right]
$$

In fact, the expression (2.1.6) can only get the approximate value for it simplifies the fractional differential.

For two-dimensional digital image, as we known, the shortest changing distance of gray-value is one pixel, and the minimal distance for filter processing is one pixel too. The method of airspace filter is moving the mask on the image one pixel each time. Provided the image to be processed has $x$ pixels and $y$ pixels in the negative $x$ and $y$ coordinate respectively. Therefore the biggest divided number on for (2.1.4) is $N \mathrm{x}=\mathrm{x} / 1=\mathrm{x}$ and $N \mathrm{y}=\mathrm{y} / 1=\mathrm{y}$. 
From the discussion above, fractional partial differential on $X$ and $Y$ coordinate can be expressed as:

$$
\begin{aligned}
& \frac{\partial^{v} f(x, y)}{\partial x^{v}} \cong\left(\frac{v}{4}+\frac{v^{2}}{8}\right) f(x+1, y)+\left(1-\frac{v^{2}}{2}-\frac{v^{3}}{8}\right) f(x, y)+\frac{1}{\Gamma(-v)} \times \\
& \sum_{k=1}^{n-2}\left[\frac{\Gamma(k-v+1)}{(k+1) !} \times\left(\frac{v}{4}+\frac{v^{2}}{8}\right)+\frac{\Gamma(k-v)}{k !} \times\left(1-\frac{v^{2}}{4}\right)+\frac{\Gamma(k-v-1)}{(k-1) !} \times\left(-\frac{v}{4}+\frac{v^{2}}{8}\right)\right] \\
& \times f(x-k, y) \\
& +\cdots+\frac{\Gamma(n-v-1)}{(n-1) ! \Gamma(-v)}\left(-\frac{v}{4}+\frac{v^{2}}{8}\right) f(x-n, y) \\
& \frac{\partial^{v} f(x, y)}{\partial y^{v}} \cong\left(\frac{v}{4}+\frac{v^{2}}{8}\right) f(x, y+1)+\left(1-\frac{v^{2}}{2}-\frac{v^{3}}{8}\right) f(x, y)+\frac{1}{\Gamma(-v)} \times \\
& \sum_{k=1}^{n-2}\left[\frac{\Gamma(k-v+1)}{(k+1) !} \times\left(\frac{v}{4}+\frac{v^{2}}{8}\right)+\frac{\Gamma(k-v)}{k !} \times\left(1-\frac{v^{2}}{4}\right)+\frac{\Gamma(k-v-1)}{(k-1) !} \times\left(-\frac{v}{4}+\frac{v^{2}}{8}\right)\right] \\
& \times f(x, y-k) \\
& +\cdots+\frac{\Gamma(n-v-1)}{(n-1) ! \Gamma(-v)}\left(-\frac{v}{4}+\frac{v^{2}}{8}\right) f(x, y-n)
\end{aligned}
$$

The corresponding fractional differential $3 \times 3$ masks on the $X$ and $Y$ coordinate can be captured easily from the expressions of (2.1.7) and (2.1.8), which are shown in Figure 2.

\begin{tabular}{|c|c|c|}
\hline 0 & $\frac{v}{4}+\frac{v^{2}}{8}$ & 0 \\
\hline 0 & $1-\frac{v^{2}}{2}-\frac{v^{3}}{8}$ & 0 \\
\hline 0 & $-\frac{5 v}{4}+\frac{5 v^{3}}{16}+\frac{v^{4}}{16}$ & 0 \\
\hline
\end{tabular}

$X$

\begin{tabular}{|c|c|c|}
\hline 0 & 0 & 0 \\
\hline$\frac{v}{4}+\frac{v^{2}}{8}$ & $1-\frac{v^{2}}{2}-\frac{v^{3}}{8}$ & $-\frac{5 v}{4}+\frac{5 v^{3}}{16}+\frac{v^{4}}{16}$ \\
\hline 0 & 0 & 0 \\
\hline
\end{tabular}

Y

Figure 2.

When $0<v<1$, it is fractional differential. According to the Figure 2, we could find the fractional differential mask is a sparse matrix, and its coefficients are nonzero, which indicates that response values of pixels are not zero in plain regions where intensity variations are negligible. We can say that fractional differential would enhance blurry regions of images to some extent.

\subsection{Multiple Scale-Space Method}

It is generally recognised that Harris algorithm can detect real corners under large scales, but the positioning of corners is not accurate. Instead, under small scales, corners positioning is accurate, but false corners ratio increases. A multiple scale-space method combing the feature information under different scales is put forward to improve the detection results. This method improves the autocorrelation matrix $M$ and adopts double discriminants to determine the corners.

Scale space theory is based on scale transformation of the original image, and its goal is to obtain scale space sequences of the image under different scale-space, then realize the edge and corner detection and feature extraction at different resolutions. 
Kernel function under scale-space is defined as:

$$
f_{\text {out }}=k \otimes f_{\text {in }}
$$

$f_{\text {in }}$ represents any signal, and $\otimes$ represents the usual convolution transform. If the maximum of $f_{\text {out }}$ is smaller than the maximum of $f_{i n}$, then $k$ is regarded as scale-space kernel.

Gaussian kernel has the property of translation-invariant, rotation-invariant and so on, and it can be proved that Gaussian kernel is the only transformation kernel to realize scale-invariant. Gaussian kernel is defined as:

$$
G(x, \sigma)=\frac{1}{\sqrt{2 \pi \sigma}} \exp \left[-\frac{x^{2}}{2 \sigma^{2}}\right]
$$

Scale space representation is obtained from Gaussian filter, and it can be expressed as $(x, \sigma)$ space. $x$ and $\sigma$ represent location parameter and scale parameter respectively.

This multiple scale-space method is based on Gaussian theory. The key step of multiple scale-space method is to change the autocorrelation matrix $M$, and fitness scaling is added to $M$. And the improved $M$ is defined as:

$$
\begin{aligned}
& M=u\left(x, y, \sigma_{1}, \sigma_{D}\right)=\sigma_{D}^{2} \times w_{1} \otimes\left[\begin{array}{cc}
I_{x}^{2} \otimes \omega_{D} & I_{x} I_{y} \otimes \omega_{D} \\
I_{x} I_{y} \otimes \omega_{D} & I_{y}^{2} \otimes \omega_{D}
\end{array}\right] \\
& =\sigma_{D}^{2} \times e^{-\left(u^{2}+v^{2}\right) / 2 \sigma_{1}^{2}} \otimes\left[\begin{array}{cc}
I_{x}^{2} \otimes e^{-\left(u^{2}+v^{2}\right) / 2 \sigma_{D}^{2}} & I_{x} I_{y} \otimes e^{-\left(u^{2}+v^{2}\right) / 2 \sigma_{D}^{2}} \\
I_{x} I_{y} \otimes e^{-\left(u^{2}+v^{2}\right) / 2 \sigma_{D}^{2}} & I_{y}^{2} \otimes e^{-\left(u^{2}+v^{2}\right) / 2 \sigma_{D}^{2}}
\end{array}\right]
\end{aligned}
$$

Where $\sigma_{1}$ and $\sigma_{D}$ represent, respectively, the integration and differentiation scales, the operator $\otimes$ represents the usual convolution. $\sigma_{1}=k \sigma_{D}, k$ represents the linear coefficient.

Here we need to compute the corner response function of each points, the corner response function of multiple scale-space method is the same as the one in Harris algorithm.

If the response function value $R$ of a point $(x, y)$ is larger than a given threshold and $R$ is a local maximum, then the point $(x, y)$ is regarded as a corner point. The local maximum is the corner response function value of image points including points under the same scale-space and the same points under different scale-space.

\section{Experimental Result and Analysis}

In this section, we are going to compare the improved algorithm with the original Harris algorithm. This section is divided into three parts. In part 1, comparisons are made between original algorithm and improved algorithm (using fractional differential mask). The fractional mask improves Harris algorithm to detect image corners with a lot of detailed information. In part 2, images have been tested and compared using original algorithm and improved algorithm (using multiple scale-space method). Finally an image is tested to illustrate that the two aspects of the improvements can well combined in part 3.

\subsection{Detection Results of Fractional Differential Mask}

As discussed in section 2.1, the coefficients of fractional differential mask are functions of order $v, v$ is taken different value to test the detection results. In this section, $v$ is adopted 0.5 and 0.7 to compare the results of fractional differential mask. Besides, two important criteria to evaluate the improved algorithm have been considered, which are the amount of real corners and accuracy. An image with detailed information have been tested to compare fractional differential mask with Prewitt mask under the same threshold, and then quantitative analysis about Figure 3 is shown in Table 1, which is used to illustrate the results in detail. 


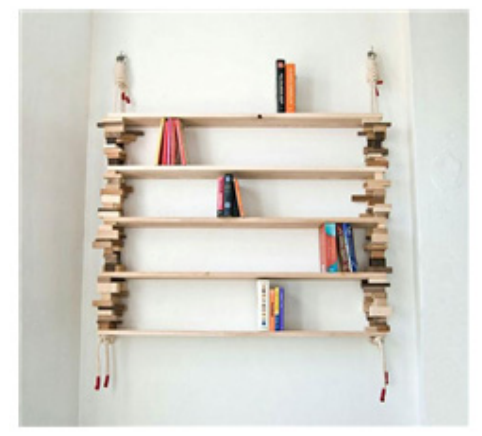

(a)

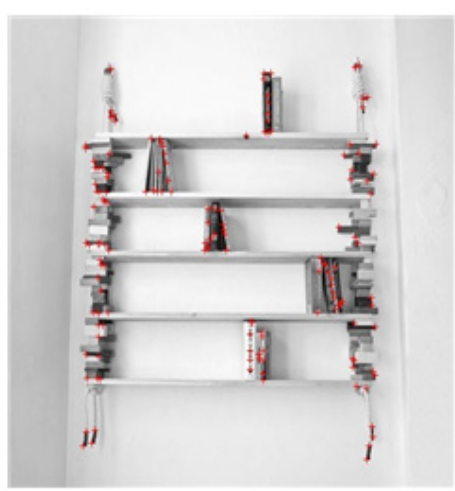

(c)

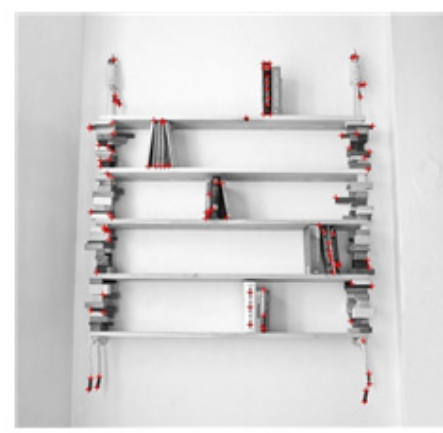

(b)

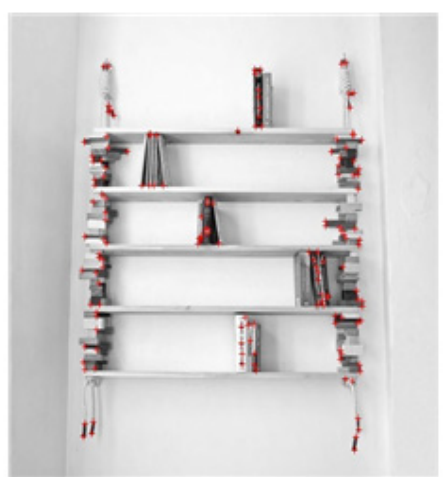

(d)

Figure 3. Detection results with different mask (from left to right: a. the original image; b. the original algorithm using Prewitt operator; c. fractional differential mask with $v=0.5$; d. fractional differential mask with $v=0.7$ )

Table 1. Quantitative analysis about Figure 3

\begin{tabular}{ccccc}
\hline algorithm & Detected corners & Real corners & False corners & accuracy \\
\hline Original algorithm & 103 & 71 & 32 & 0.69 \\
Improved algorithm $(v=0.5)$ & 138 & 97 & 41 & 0.71 \\
Improved algorithm $(v=0.7)$ & 149 & 115 & 34 & 0.77 \\
\hline
\end{tabular}

As shown in Table 1, we could find that Harris algorithm detection result is good when dealing with regions with obvious gray-value changes, and a large proportion of corners are detected by the original algorithm. But it is also obvious that some corners are ignored in area where is corrupted by noises or textures. While using fractional differential algorithm, many more real corners are detected, even in blurred edges areas. From Table 1, we could also find the main shortcoming of improved algorithm is that it obtains more false corners. To some extent, the shortcoming is the negative effects of texture enhancements. As a whole, the detection accuracy of improved algorithm is higher than the original one. Therefore, we can say that the improved algorithm can well enhance texture details and obtains better detection results than the original algorithm.

\subsection{Detection Results of Multiple Scale-Space Method}

In order to verify the detection result of multiple scale-space method, experiments have been carried out on an real-world image. We get different detection results by changing the value of $\sigma$, which are shown in Figure 4 . 


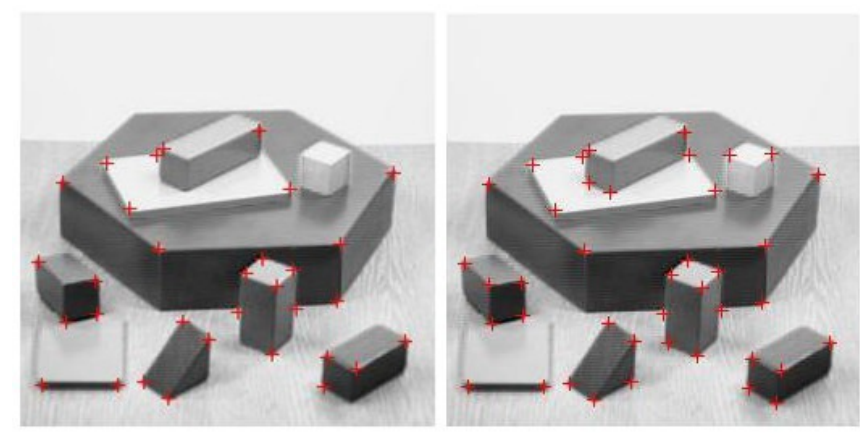

(a)

(b)

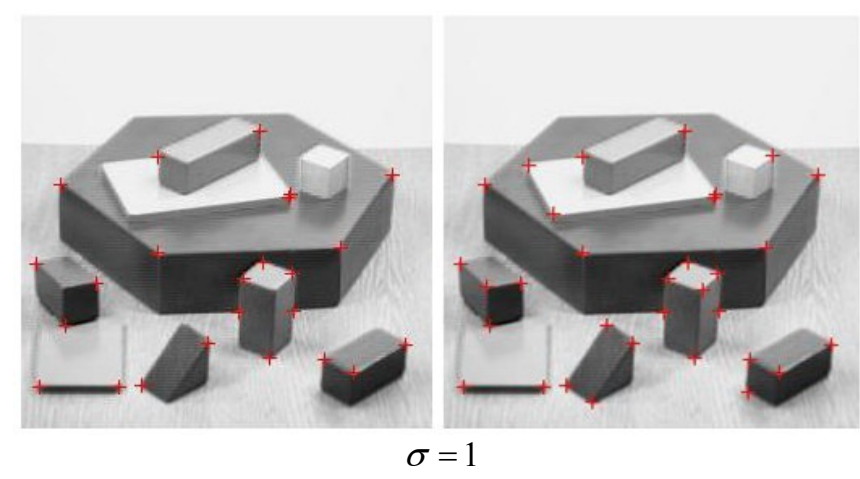

(a)

(b)

Figure 4. Detection results between Harris algorithm (a) and improved algorithm (b)

It can be concluded from Figure 4 that the improved algorithm can detect more accurate corners than Harris algorithm when $\sigma$ changes. The original algorithm detects less corners when the scale parameter get smaller, while the improved algorithm can preserve a good detection result.

\subsection{Combination of two Improved Aspects}

Another image is tested to illustrate the better detection results of the improved algorithm combined with two improved aspects $(v=0.7)$, and the result is shown in Figure 5.

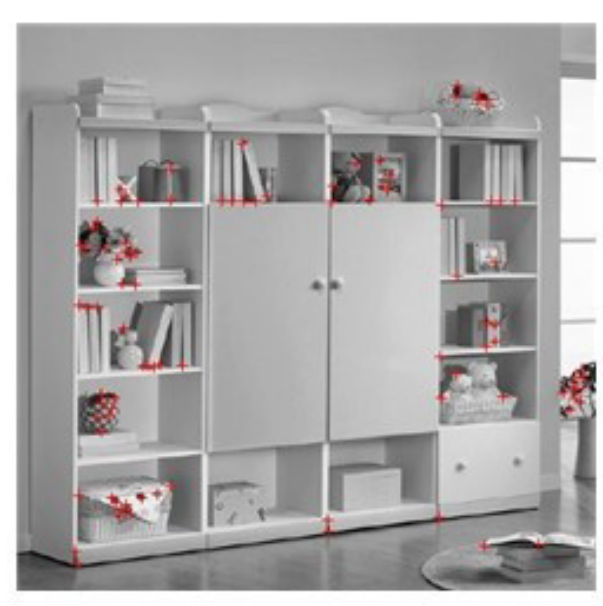

(a) corner detection result of Harris algorithm

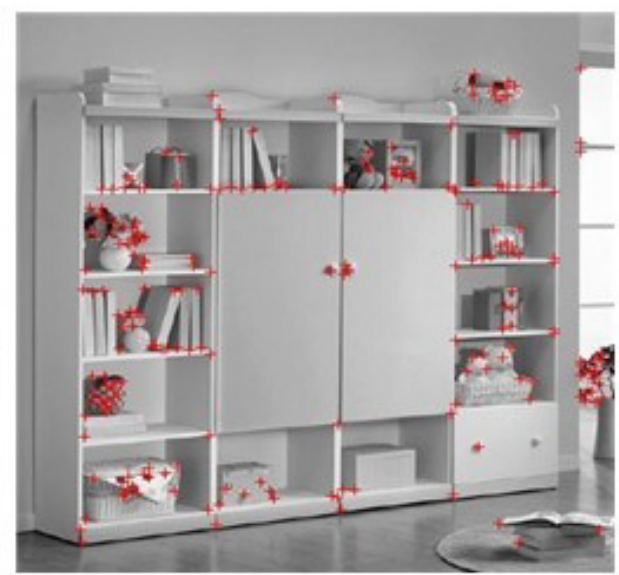

(b) corner detection result of improved algorithm

Figure 5. Corner detection results of Harris algorithm and improved algorithm 
Table 2. Quantitative analysis about Figure 5

\begin{tabular}{ccccc}
\hline algorithm & Detected corners & Real corners & False corners & accuracy \\
\hline Harris algorithm & 174 & 76 & 98 & 0.44 \\
Improved algorithm & 225 & 120 & 105 & 0.53 \\
\hline
\end{tabular}

As shown in Table 2, we could find many more corners could be detected by the improved algorithm, and the detection accuracy of improved algorithm is higher than the Harris algorithm. In a word, the improved algorithm could obtain better detection result when dealing with images with a lot of detailed informations.

\section{Conclusion}

In this paper, firstly, Harris algorithm has been introduced. And then two improvements on Harris algorithm are put forward: one is the fractional differential mask, and the other is multiple scale-space method. Finally experiments are carried out to illustrate the detection results of improved algorithm, which indicate that the improved algorithm is better than the Harris algorithm.

\section{References}

Bellavia, F., Tegolo, D., \& Valenti, C. (2011). Improving Harris corner selection strategy. IET Computer Vision, 5(2), 87-96. http://dx.doi.org/10.1049/iet-cvi.2009.0127

Chen, B. F., \& Cai, Z. X. (2005). Harris corner detection based on multiple scale-space theory. Journal of central south university (China), 36(5), 741-754.

Davis, L. S., \& Freeman, H. (1997). A corner finding algorithm for chain coded curves. IEEE Trans. on Compt, 26(3), 297-303. http://dx.doi.org/10.1109/TC.1977.1674825

Huang, M., Yang Z. Z., \& Zhou, J. L. (2008). Edges detection based on fractional differential. Journal of Si Chuan university (China), 40(1), 152-157.

Mathieu, B., Melchior, P., \& Oustaloup, A. (2003). Fractional differentiation for edge detection. Signal Processing, 83(11), 2421-2432. http://dx.doi.org/10.1016/S0165-1684(03)00194-4

Park, H., Park, J., \& Ryu, J. B. (2011). Corner classification using Harris algorithm. Electronics letters, 47(9), 536-538. http://dx.doi.org/10.1049/el.2011.0594

Pu, Y. F., Yuan, X., \& Zhou, J. L. (2010). Fractional differential mask:a fractional differential-based approach for multiscale texture enhancement. IEEE Transactions on Image processing, 19(2), 491-511. http://dx.doi.org/10.1109/TIP.2009.2035980

Wu, J. H., Zhang, L., \& Zhou, N. R. (2010). Image encryption based on the multiple-order discrete fractional $\begin{array}{llll}\text { cosine } \quad \text { Optics } & \text { Communications, } & \text { 283(9), }\end{array}$ http://dx.doi.org/10.1016/j.optcom.2009.12.066

Zhang, X. H., \& Yang, D. (2009). A novel Harris multi-scale corner detection algorithm. Computer application(China), 29(3), 1-5.

\section{Copyrights}

Copyright for this article is retained by the author(s), with first publication rights granted to the journal.

This is an open-access article distributed under the terms and conditions of the Creative Commons Attribution license (http://creativecommons.org/licenses/by/3.0/). 